\title{
Screening of Mungbean (Vigna radiata L. Wilczek) Genotypes against Water Stress Mediated through Polyethylene Glycol
}

\author{
L. Swathi ${ }^{1 *}$, D.M. Reddy ${ }^{1}$, P. Sudhakar ${ }^{2}$ and V. Vineela ${ }^{2}$ \\ ${ }^{1}$ Department of Genetics and Plant Breeding, S.V. Agricultural College, Tirupati 517502, A P, India \\ ${ }^{2}$ Department of Crop physiology, S.V. Agricultural College, Tirupati 517502, A P, India \\ *Corresponding author
}

\section{A B S T R A C T}

\section{Keywords}

Mungbean, PEG,

Germination

percentage, Root

length, Shoot

length, Vigour

index.

Article Info

Accepted:

21 September 2017

Available Online:

10 October 2017
Thirty one mungbean (Vigna radiata L. Wilczek) genotypes were screened for drought tolerance under laboratory conditions in CRD using PEG 6000 at different concentrations of $-0.3 \mathrm{MPa},-0.6 \mathrm{MPa}$ and $-0.9 \mathrm{MPa}$. The genotypes showed reduction in germination percentage and shoot length and increase in root length under water stress condition. Screening of genotypes based on single morphological parameter appears to be limiting due to inconsistency in growth responses of different seedling parts. However, vigour index is a stable character and can be considered as a useful tool to screen drought tolerant genotypes. Besides, non-parametric selection for yield and drought tolerance was scored based on the mean performance of each character. Based on vigour index and nonparametric selection, the genotype ML 267 was found to be drought tolerant with high yield.

\section{Introduction}

Plants are subjected to various abiotic stresses like drought, salinity; heat, temperature; cold and water logging have adverse effect on crop productivity and causes severe agriculture losses predominantly in arid and semi-arid tropic region like India (Abdul et al., 2006) and (Rodriguez et al., 2005). Of these stresses, drought is one of the major abiotic stresses which limits the crop production. Water stress limits plant survival and early seedling growth by delaying its beginning or decreasing the final germinability (Gamze et al., 2005). Seed germination and early seedling growth are considered as most critical phases for seed establishment, determining successful crop production. Mungbean (Vigna radiata. $\mathrm{L}$ ) is a major pulse crop of Asia after chickpea and pigeonpea.

It is a short crop duration possessing qualities like nitrogen fixing ability, inhibition of soil erosion, soil enrichment, low input requirements and wide adaptability. Mungbean is source of high quality protein, rich in phosphorus, calcium and vitamins. Unfortunately, available mungbean varieties lack satisfactory level of drought tolerance and at present drought tolerant high yielding varieties are not available in this crop. One of the elements of overcoming the stress is to 
develop new varieties. However, drought tolerance has a complex nature which makes this problem difficult to solve and involves different approaches and methods. The process of creating new varieties could be facilitated by screening of genotypes with higher resistance. Selection of parents with a better drought tolerance is critical in dry environments (Ashraf et al., 1992; Tuberosa and Salvi, 2006).

However, controlled and uniformly repeated simulation of drought in the field cannot be easily achieved (Shaheen and Hood-Nowotny, 2005). In-vitro drought screening methods facilitates progress in our understanding of drought resistance. The high molecular weight substance polyethylene glycol (PEG) causes osmotic shock which is one of the components of the drought. Three different concentrations (-0.3 $\mathrm{MPa},-0.6 \mathrm{MPa}$ and -0.9 $\mathrm{MPa})$ of PEG were used in our experiment to identify drought tolerant genotypes based on vigour index.

\section{Materials and Methods}

The experiment was conducted in the laboratory of the department of Genetics and Plant Breeding, Sri Venkateswara Agricultural College, Tirupati using thirty one mungbean genotypes obtained from Regional Agricultural Research Station, Lam, Guntur and Agricultural Research Station, Madhira. The experiment was conducted in CRD with two replications. Thirty seeds $(10$ per petridish) from each genotype were surface sterilized with $0.1 \%(\mathrm{w} / \mathrm{v})$ Mercuric chloride for three minutes and washed thoroughly with distilled water. External water potentials of $0.3,-0.6$ and $-0.9 \mathrm{MPa}$ were prepared by using Poly Ethylene Glycol (PEG) 6000 as per method described by Michel and Kaufmann (1973). The seeds were placed in Petri-dishes moistened with each four water potential treatments viz., 0.0 (control), -0.3, -
0.6 and $-0.9 \mathrm{MPa}$ after soaking for five hours in their respective concentrations in two replications at room temperature. Presoaked seeds were allowed to develop seedlings in their respective concentration of PEG for six days following standard glass plate technique (Nandi and Bera, 1995). After six days seedlings were removed from the petridishes and germination percentage, root length and shoot length were recorded, from which the vigour index was calculated using the following formula.

Vigour index $=[$ root length $(\mathrm{cm})+$ shoot length $(\mathrm{cm})] *$ Germination percentage $(\%)$

Besides, non-parametric selections for yield, drought tolerance were scored based on the mean performance of each character. The genotypes having higher mean, than the sum of grand mean and standard error of deviation were scored as ' 1 ' and the genotypes with lower mean values were scored as ' 0 '. The scores for all the characters along with yield of each genotype is summed up to identify the drought tolerant variety.

\section{Results and Discussion}

The mean data obtained on different parameters were subjected to ANOVA (results not presented) and significant differences were observed for all the characters (germination percentage, root length, shoot length and vigour index) at various concentrations, indicating the existence of wide variability for all the characters. The mean performance of the genotypes for germination, root length and shoot length were presented in table 1 . There was gradual decrease in germination percentage and shoot length with increased concentration of PEG. The mean performance of germination percentage and shoot length decreased when compared with the control as the concentration of PEG was increased. 
Table.1 Mean performance of 31 mungbean genotypes at various concentrations of PEG

\begin{tabular}{|c|c|c|c|c|c|c|c|c|c|c|c|c|c|c|c|c|c|}
\hline \multirow[b]{2}{*}{$\begin{array}{l}\text { Sl. } \\
\text { No. }\end{array}$} & \multirow[b]{2}{*}{ Genotype } & \multicolumn{4}{|c|}{ Germination \% } & \multicolumn{4}{|c|}{ Shoot length $(\mathrm{cm})$} & \multicolumn{4}{|c|}{ Root length $(\mathrm{cm})$} & \multicolumn{4}{|c|}{ Vigour index } \\
\hline & & Control & $\begin{array}{c}-0.3 \\
\text { MPa }\end{array}$ & $\begin{array}{c}-0.6 \\
\text { MPa }\end{array}$ & $\begin{array}{c}-0.9 \\
\text { MPa }\end{array}$ & Control & $\begin{array}{c}-\mathbf{- 0 . 3} \\
\text { MPa }\end{array}$ & $\begin{array}{c}-0.6 \\
\text { MPa }\end{array}$ & $\begin{array}{l}-0.9 \\
\text { MPa }\end{array}$ & Control & $\begin{array}{c}-0.3 \\
\text { MPa }\end{array}$ & $\begin{array}{c}-0.6 \\
\text { MPa }\end{array}$ & $\begin{array}{c}-0.9 \\
\text { MPa }\end{array}$ & Control & $\begin{array}{c}-0.3 \\
\text { MPa }\end{array}$ & $\begin{array}{c}-0.6 \\
\text { MPa }\end{array}$ & $\begin{array}{c}-0.9 \\
\text { MPa }\end{array}$ \\
\hline 1. & GIVT 203 & 100.00 & 100.00 & 95.00 & 85.00 & 15.15 & 13.55 & 3.96 & 0.70 & 9.25 & 7.25 & 6.03 & 6.94 & 2440.00 & 2080.00 & 1026.00 & 650.00 \\
\hline 2. & KM-8-657 & 100.00 & 85.00 & 75.00 & 60.00 & 15.42 & 7.90 & 2.43 & 0.42 & 9.00 & 6.30 & 5.73 & 8.02 & 2442.50 & 1210.00 & 578.20 & 356.40 \\
\hline 3. & KM-122 & 100.00 & 100.00 & 75.00 & 0.00 & 15.55 & 7.95 & 1.29 & 0.00 & 8.85 & 7.18 & 4.11 & 0.00 & 2440.00 & 1513.00 & 416.00 & 0.00 \\
\hline 4. & ASHA & 100.00 & 95.00 & 90.00 & 75.00 & 15.29 & 6.92 & 5.15 & 0.80 & 7.38 & 5.45 & 6.89 & 8.00 & 2267.00 & 1170.40 & 1252.00 & 664.10 \\
\hline 5. & WGG-37 & 100.00 & 90.00 & 85.00 & 75.00 & 13.76 & 9.20 & 7.37 & 0.79 & 7.02 & 7.50 & 8.96 & 6.02 & 2079.00 & 1503.00 & 1393.60 & 508.50 \\
\hline 6. & PUSA 9531 & 100.00 & 90.00 & 70.00 & 80.00 & 17.26 & 3.93 & 1.99 & 1.00 & 7.92 & 6.42 & 7.96 & 8.78 & 2519.00 & 931.95 & 717.50 & 782.00 \\
\hline 7. & COGG 974 & 100.00 & 85.00 & 60.00 & 70.00 & 17.54 & 4.67 & 1.95 & 0.63 & 7.64 & 7.04 & 8.20 & 8.90 & 2518.00 & 996.10 & 607.20 & 667.10 \\
\hline 8. & LGG 528 & 100.00 & 90.00 & 95.00 & 85.00 & 14.32 & 11.35 & 2.01 & 1.80 & 7.10 & 7.94 & 8.08 & 7.99 & 2142.00 & 1736.55 & 956.00 & 834.10 \\
\hline 9. & VG 7098A & 100.00 & 100.00 & 95.00 & 75.00 & 17.45 & 8.00 & 5.08 & 0.71 & 8.89 & 6.10 & 9.02 & 9.55 & 2634.00 & 1410.00 & 1315.80 & 733.00 \\
\hline 10. & EC- 396117 & 100.00 & 85.00 & 80.00 & 0.00 & 11.41 & 1.49 & 1.22 & 0.00 & 7.28 & 4.08 & 2.90 & 0.00 & 1869.00 & 476.40 & 363.20 & 0.00 \\
\hline 11. & WGG 2 & 100.00 & 95.00 & 90.00 & 95.00 & 14.14 & 3.77 & 0.70 & 0.55 & 10.18 & 5.05 & 3.85 & 5.75 & 2432.00 & 835.00 & 324.00 & 554.20 \\
\hline 12. & VG- 6197A & 75.00 & 75.00 & 60.00 & 70.00 & 12.00 & 10.09 & 2.37 & 0.61 & 6.16 & 8.07 & 4.70 & 7.54 & \begin{tabular}{|l|}
1361.60 \\
\end{tabular} & 1367.55 & 390.60 & 570.50 \\
\hline 13. & LGG 460 & 100.00 & 85.00 & 85.00 & 0.00 & 13.18 & 0.93 & 1.68 & 0.00 & 7.33 & 4.91 & 5.76 & 0.00 & 2051.50 & 496.75 & 560.00 & 0.00 \\
\hline 14. & LGG 450 & 100.00 & 90.00 & 95.00 & 0.00 & 15.60 & 11.69 & 1.04 & 0.00 & 8.21 & 8.71 & 7.00 & 0.00 & 2381.00 & 1836.00 & 792.00 & 0.00 \\
\hline 15. & LGG 407 & 100.00 & 90.00 & 82.50 & 0.00 & 14.77 & 10.99 & 1.09 & 0.00 & 8.06 & 6.71 & 4.85 & 0.00 & 2283.50 & 1593.45 & 542.30 & 0.00 \\
\hline 16. & LGG 410 & 100.00 & 100.00 & 75.00 & 70.00 & 15.55 & 6.75 & 2.28 & 0.84 & 9.07 & 5.70 & 6.68 & 12.11 & 2462.50 & 1245.00 & 702.40 & 801.50 \\
\hline 17. & TM 96-2 & 100.00 & 75.00 & 60.00 & 45.00 & 16.08 & 8.05 & 6.50 & 1.09 & 8.80 & 6.95 & 7.24 & 5.44 & 2488.00 & 1123.90 & 637.00 & 136.65 \\
\hline
\end{tabular}

\begin{tabular}{|c|c|c|c|c|c|c|c|c|c|c|c|c|c|c|c|c|c|}
\hline \multirow[b]{2}{*}{$\begin{array}{l}\text { Sl. } \\
\text { No. }\end{array}$} & \multirow[b]{2}{*}{ Genotype } & \multicolumn{4}{|c|}{ Germination \% } & \multicolumn{4}{|c|}{ Shoot length $(\mathrm{cm})$} & \multicolumn{4}{|c|}{ Root length $(\mathrm{cm})$} & \multicolumn{4}{|c|}{ Vigour index } \\
\hline & & Control & $\begin{array}{c}-0.3 \\
\text { MPa }\end{array}$ & $\begin{array}{c}-0.6 \\
\text { MPa }\end{array}$ & $\begin{array}{l}-0.9 \\
\mathrm{MPa}\end{array}$ & Control & $\begin{array}{c}-0.3 \\
\text { MPa }\end{array}$ & $\begin{array}{c}-0.6 \\
\text { MPa }\end{array}$ & $\begin{array}{l}-0.9 \\
\mathrm{MPa}\end{array}$ & Control & $\begin{array}{c}-0.3 \\
\text { MPa }\end{array}$ & $\begin{array}{c}-0.6 \\
\text { MPa }\end{array}$ & \begin{tabular}{|l|}
-0.9 \\
MPa
\end{tabular} & Control & $\begin{array}{c}-0.3 \\
\text { MPa }\end{array}$ & $\begin{array}{c}-0.6 \\
\text { MPa }\end{array}$ & \begin{tabular}{|l|}
-0.9 \\
MPa
\end{tabular} \\
\hline 18. & TLM-7 & 100.00 & 100.00 & 85.00 & 60.00 & 17.26 & 7.96 & 3.94 & 0.52 & 10.10 & 6.16 & 8.69 & 10.77 & \begin{tabular}{|l|}
2736.50 \\
\end{tabular} & 1412.00 & 971.20 & 677.70 \\
\hline 19. & AKM 9904 & 100.00 & 100.00 & 75.00 & 80.00 & 17.60 & 7.48 & 2.83 & 1.02 & 9.31 & 7.22 & 9.74 & 11.33 & 2691.00 & 1470.00 & 821.80 & 988.00 \\
\hline 20. & RMG 492 & 100.00 & 90.00 & 80.00 & 55.00 & 15.04 & 1.66 & 1.15 & 0.86 & 7.40 & 5.53 & 5.11 & 11.44 & 2244.50 & 647.10 & 510.40 & 681.50 \\
\hline 21. & PUSA VISHAL & 100.00 & 100.00 & 85.00 & 75.00 & 15.06 & 3.50 & 1.55 & 0.98 & 7.13 & 5.46 & 8.94 & 11.15 & 2219.00 & 896.00 & 774.40 & 911.50 \\
\hline 22. & MGG350 & 100.00 & 85.00 & 70.00 & 85.00 & 14.95 & 5.14 & 1.95 & 0.96 & 7.85 & 7.81 & 9.94 & 11.63 & 2280.50 & 1100.60 & 792.40 & 1066.30 \\
\hline 23. & ML145 & 100.00 & 95.00 & 85.00 & 70.00 & 16.10 & 2.32 & 1.72 & 0.51 & 11.57 & 7.23 & 7.31 & 11.55 & \begin{tabular}{|l|}
2767.00 \\
\end{tabular} & 912.70 & 637.60 & 494.20 \\
\hline 24. & IPM-02-03 & 100.00 & 85.00 & 95.00 & 65.00 & 12.39 & 4.26 & 1.66 & 0.79 & 8.14 & 8.59 & 8.08 & 9.94 & 2053.00 & 1089.30 & 815.40 & 699.20 \\
\hline 25. & IPM-02-19 & 100.00 & 85.00 & 85.00 & 70.00 & 9.85 & 9.80 & 4.86 & 1.37 & 8.65 & 3.76 & 6.14 & 9.99 & \begin{tabular}{|l|}
1850.00 \\
\end{tabular} & 1154.90 & 811.20 & 654.85 \\
\hline 26. & MH-3-18 & 100.00 & 85.00 & 85.00 & 0.00 & 18.18 & 3.69 & 0.85 & 0.00 & 17.10 & 5.75 & 7.75 & 0.00 & 3528.00 & 797.20 & 625.60 & 0.00 \\
\hline 27. & ML-267 & 100.00 & 95.00 & 65.00 & 95.00 & 19.00 & 15.85 & 7.54 & 0.85 & 8.90 & 6.10 & 10.96 & 14.14 & \begin{tabular}{|l|}
2790.00 \\
\end{tabular} & 2088.70 & 1156.80 & 1425.45 \\
\hline 28. & MH-565 & 100.00 & 90.00 & 100.00 & 100.00 & 18.14 & 8.09 & 0.96 & 0.45 & 7.91 & 6.85 & 9.71 & 10.89 & \begin{tabular}{|l|}
2605.00 \\
\end{tabular} & 1341.60 & 1024.00 & 1133.50 \\
\hline 29. & PM110 & 100.00 & 100.00 & 95.00 & 0.00 & 17.90 & 7.99 & 0.88 & 0.00 & 10.20 & 7.80 & 9.91 & 0.00 & \begin{tabular}{|l|}
2810.00 \\
\end{tabular} & 1579.00 & 1076.00 & 0.00 \\
\hline 30. & MGG295 & 100.00 & 65.00 & 95.00 & 95.00 & 18.16 & 9.30 & 2.86 & 0.50 & 9.00 & 3.09 & 4.38 & 5.38 & 2716.00 & 806.50 & 610.00 & 506.80 \\
\hline \multirow[t]{2}{*}{31.} & MGG347 & 100.00 & 90.00 & 85.00 & 95.00 & 13.42 & 4.20 & 2.16 & 0.98 & 7.65 & 4.95 & 6.55 & 11.67 & \begin{tabular}{|l|}
2107.00 \\
\end{tabular} & 823.50 & 644.00 & 1014.20 \\
\hline & General Mean & 99.19 & 90.16 & 82.33 & 59.03 & 15.40 & 7.04 & 2.67 & 0.63 & 8.68 & 6.37 & 7.13 & 7.25 & \begin{tabular}{|l|}
2393.81 \\
\end{tabular} & 1214.32 & 804.67 & 564.87 \\
\hline
\end{tabular}


Table.2 Non-parametric selection of mungbean genotypes based on PEG screening and yield

\begin{tabular}{|c|c|c|c|c|c|c|c|}
\hline $\begin{array}{l}\text { Sl. } \\
\text { No. }\end{array}$ & Genotypes & Germination & $\begin{array}{l}\text { Shoot } \\
\text { length }\end{array}$ & $\begin{array}{l}\text { Root } \\
\text { length }\end{array}$ & $\begin{array}{l}\text { Vigour } \\
\text { index }\end{array}$ & $\begin{array}{l}\text { Seed } \\
\text { yield }\end{array}$ & Sum \\
\hline 1 & GIVT 203 & 1 & 0 & 0 & 0 & 0 & 1 \\
\hline 2 & KM-8-657 & 0 & 0 & 0 & 0 & 0 & 0 \\
\hline 3 & KM-122 & 0 & 0 & 0 & 0 & 1 & 1 \\
\hline 4 & ASHA & 1 & 1 & 0 & 1 & 0 & 3 \\
\hline 5 & WGG-37 & 1 & 1 & 0 & 0 & 1 & 3 \\
\hline 6 & PUSA 9531 & 1 & 1 & 1 & 1 & 0 & 4 \\
\hline 7 & COGG 974 & 1 & 0 & 1 & 1 & 0 & 3 \\
\hline 8 & LGG 528 & 1 & 1 & 0 & 1 & 0 & 3 \\
\hline 9 & VG 7098A & 1 & 1 & 1 & 1 & 0 & 4 \\
\hline 10 & EC- 396117 & 0 & 0 & 0 & 0 & 0 & 0 \\
\hline 11 & WGG 2 & 1 & 0 & 0 & 0 & 1 & 2 \\
\hline 12 & VG- 6197A & 0 & 0 & 0 & 0 & 0 & 0 \\
\hline 13 & LGG 460 & 0 & 0 & 0 & 0 & 0 & 0 \\
\hline 14 & LGG 450 & 0 & 0 & 0 & 0 & 0 & 0 \\
\hline 15 & LGG 407 & 0 & 0 & 0 & 0 & 0 & 0 \\
\hline 16 & LGG 410 & 1 & 1 & 1 & 1 & 0 & 4 \\
\hline 17 & TM 96-2 & 0 & 1 & 0 & 0 & 0 & 1 \\
\hline 18 & TLM-7 & 0 & 0 & 1 & 1 & 0 & 2 \\
\hline 19 & AKM 9904 & 0 & 1 & 1 & 1 & 1 & 4 \\
\hline 20 & RMG 492 & 0 & 1 & 1 & 1 & 1 & 4 \\
\hline 21 & PUSA VISHAL & 1 & 1 & 1 & 1 & 0 & 4 \\
\hline 22 & MGG350 & 1 & 1 & 1 & 1 & 0 & 4 \\
\hline 23 & ML145 & 1 & 0 & 1 & 0 & 0 & 2 \\
\hline 24 & IPM-02-03 & 0 & 1 & 1 & 1 & 0 & 3 \\
\hline 25 & IPM-02-19 & 1 & 1 & 1 & 0 & 1 & 4 \\
\hline 26 & MH-3-18 & 0 & 0 & 0 & 0 & 0 & 0 \\
\hline 27 & ML-267 & 1 & 1 & 1 & 1 & 1 & 5 \\
\hline 28 & MH-565 & 1 & 0 & 0 & 1 & 0 & 2 \\
\hline 29 & PM110 & 0 & 0 & 0 & 0 & 1 & 1 \\
\hline 30 & MGG295 & 1 & 0 & 0 & 1 & 1 & 3 \\
\hline \multirow[t]{2}{*}{31} & MGG347 & 1 & 1 & 1 & 0 & 1 & 4 \\
\hline & General Mean & 0.548 & 0.483 & 0.451 & 0.483 & 0.322 & 2.290 \\
\hline
\end{tabular}

$0=($ Less than general mean + standard deviation $), 1=($ Greater than general mean + standard deviation $)$ 
Plate.1 In vitro screening of mungbean genotype (ML 267) at different concentrations of PEG

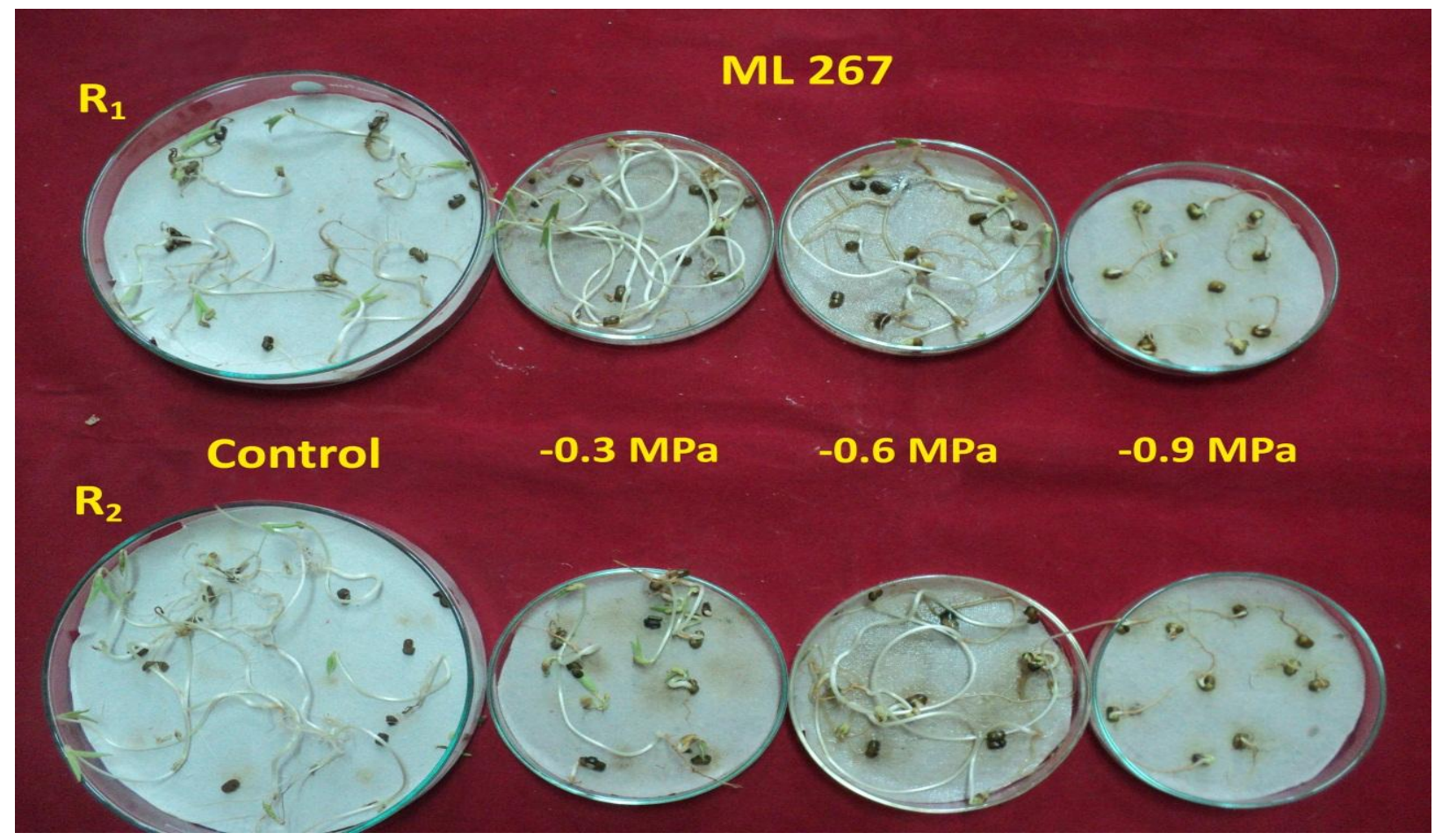

Plate.2 In vitro screening of mungbean genotypes (MH 565) at different concentrations of PEG

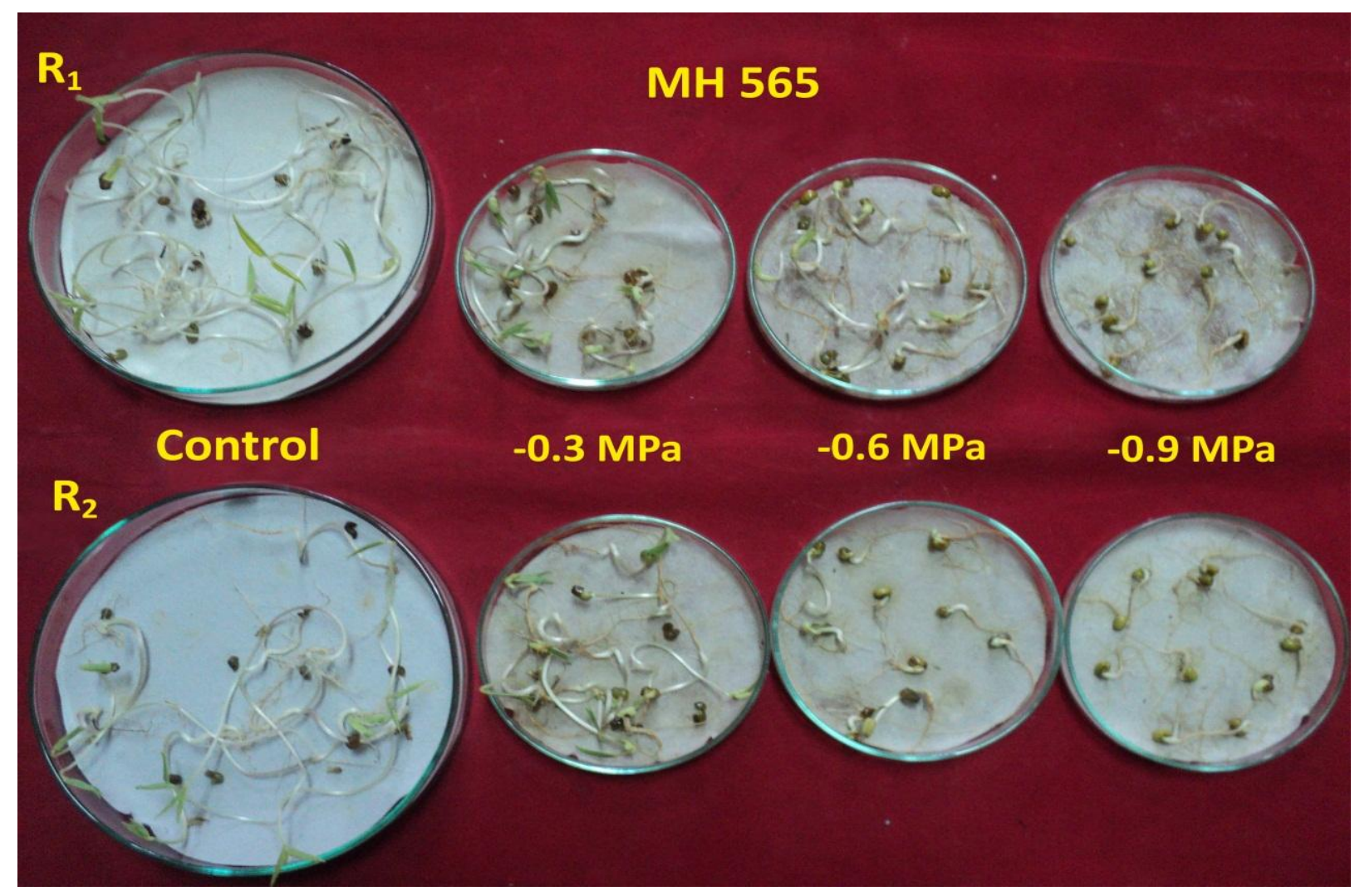


Plate.3 In vitro screening of mungbean genotypes (MGG 350) at different concentrations of PEG

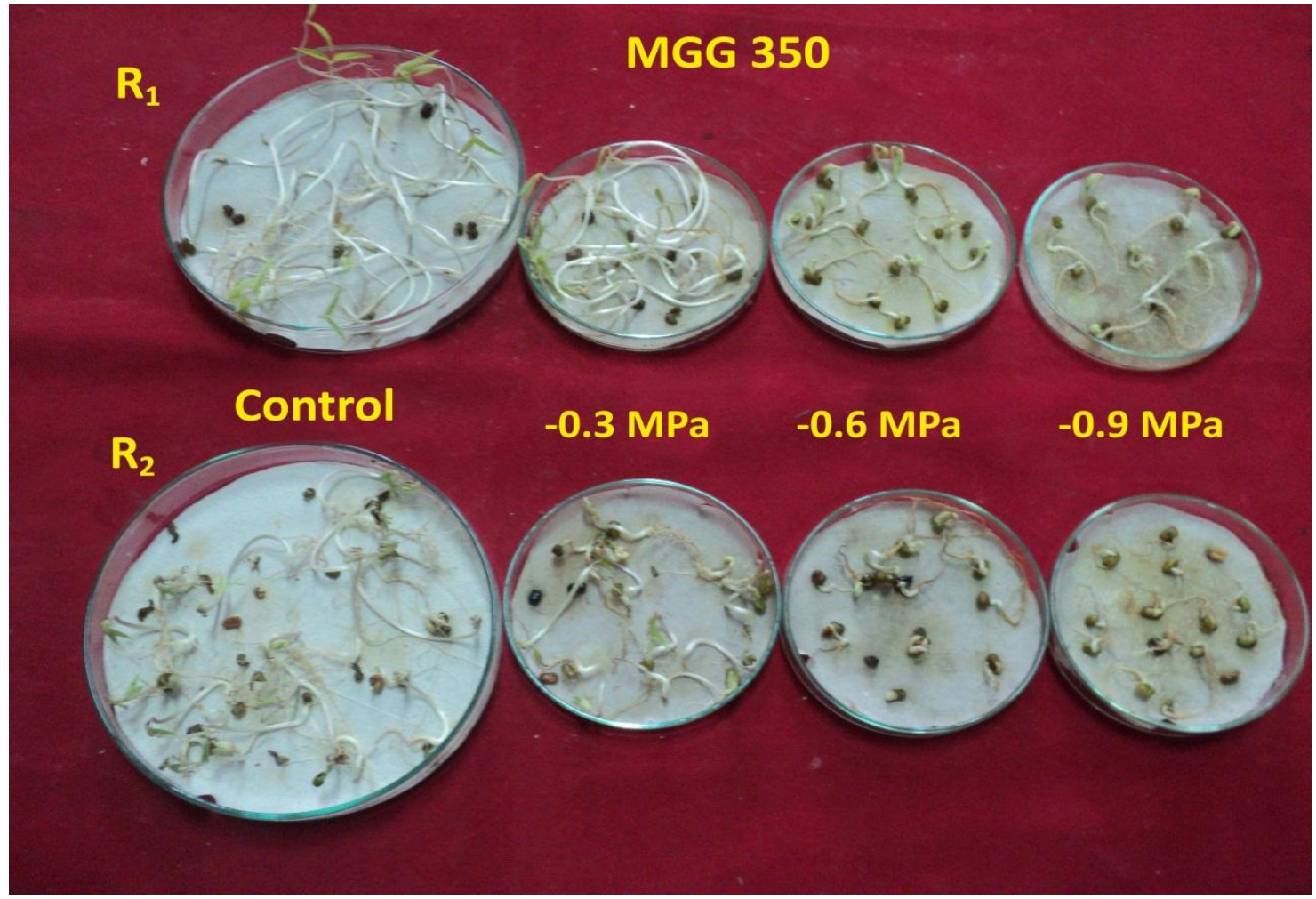

Plate.4 In vitro screening of mungbean genotypes (MGG 347) at different concentrations of PEG

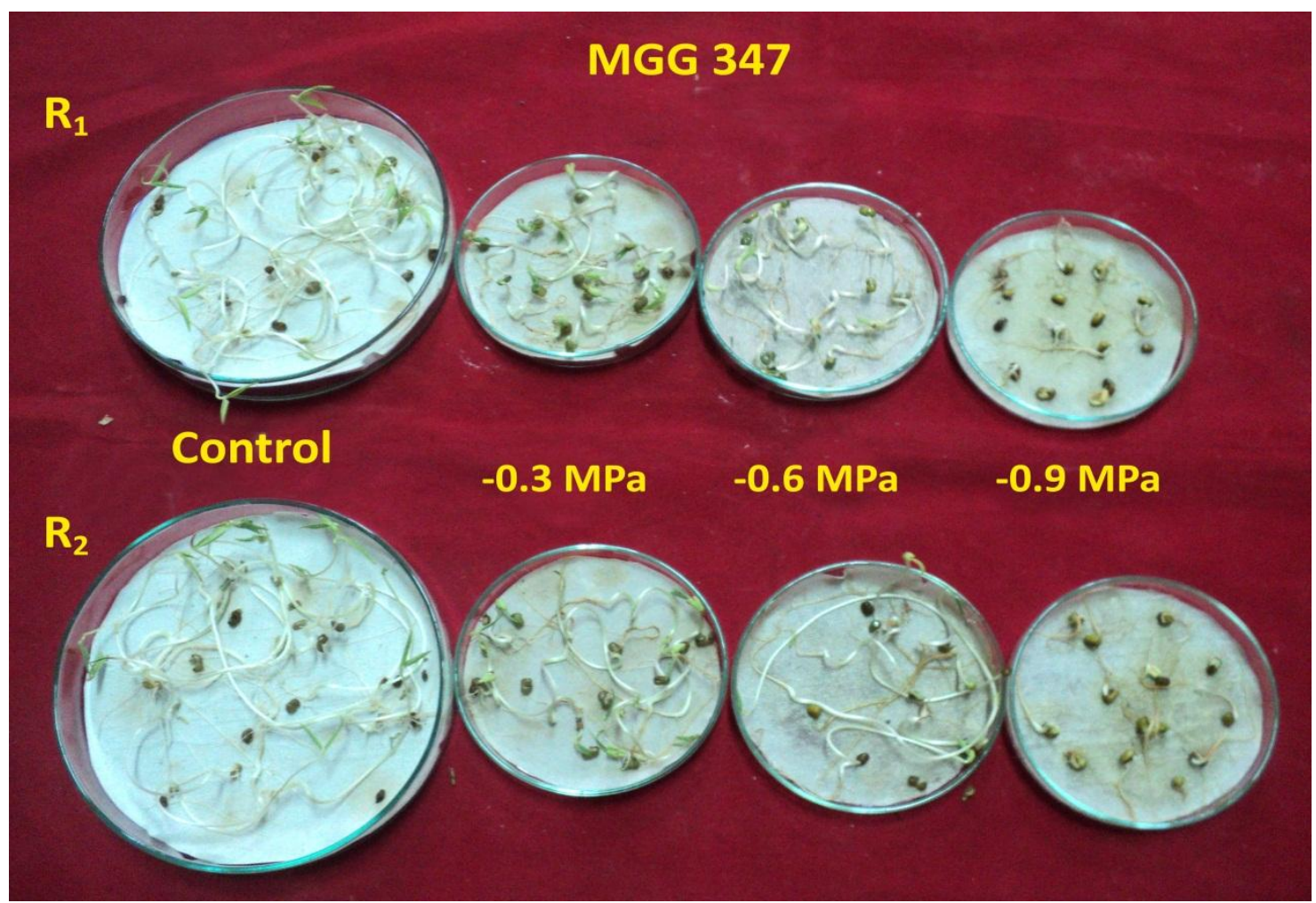


However, the root growth was increased as the concentration of PEG increased indicating it as one of the morphological adaptation to drought (Table 1). These results were also in accordance with the findings of Dutta et al., (2007) and Rajwider et al., (2011) in mungbean. The genotypes varied significantly for vigour index in all the concentrations. In reduced water potential of $-0.3 \mathrm{MPa}$ the vigour index ranged between 476.40 (EC 396117) and 2088.70 (ML 267) with an average mean of 1214.32. Similarly, at $0.6 \mathrm{MPa}$ vigour index ranged between 324.00 (WGG 2) and 1393.60 (WGG 37) with an average mean of 804.67. At $-0.9 \mathrm{MPa}$ the vigour index index ranged between 136.65 (TM 96-2) and 1425.45 (ML 267) with an average mean of 564.87. Based on the Nonparametric study, the genotype ML 267 showed the highest score (5) owing to its high yield coupled with drought tolerance (Table 2).

Thus from the above study the genotypes ML 267, MH 565, MGG 350 and MGG 347 (Plate 1 to 4) were found to be stress tolerant at high $(-0.9 \mathrm{MPa})$ concentration of PEG due to their good germination percentage along with more root and shoot lengths. In moderate stress ($0.6 \mathrm{MPa})$ condition the genotypes WGG 37, VG 7098A, ASHA and ML 267 performed well. At low stress $(-0.3 \mathrm{MPa})$ the genotypes ML 267, GIVT 203, LGG 450 and LGG 528 showed better performance. In contrast, the genotypes KM 122, EC 396117, LGG 460, LGG 450, LGG 407, MH 3-18 and PM 110 are highly susceptible to water stress (-0.3, 0.6 and -0.9) registering low germination percentage coupled with lesser shoot and root length. However, these data could be a basis for development of rapid preliminary tests for evaluation of drought tolerance of great number of genotypes under laboratory conditions simulating the stress. Since drought is a complex trait, further conformational studies has to be conducted to confirm a variety as drought tolerant before recommending to the growers of drought prone areas.

\section{Acknowledgement}

I express deep sense of gratitude to my dear friend Late Vineela vaka, who was with me throughout my research work with her intense warmth, sisterly care, affection and research related help. I would prefer to pay homage by dedicating this research to my friend.

\section{References}

Abdul, W., Ishfaq, A. M., Ghulam, Q., Ghulam, M., Tariq, M. and. Muhammad, A. 2006. Effect of salinity on germination, growth, yield, ionic balance and solute composition of Pigeonpea (Cajanus cajan (L) Millsp.). Pak. J. Bot., 38 (4): 1103-1117.

Ashraf, M., Bokhari, H. and Cristiti, S.N. 1992. Variation in osmotic adjustment of lentil (Lens culinaris, Medik) in response to drought. Acta. Bot. Neerl., 41: 51- 62.

Dutta and Bera, A. K. 2008. Screening of mungbean genotypes for drought tolerance. Legume Res., 31 (2): 145148.

Gamze, O., Mehmet, D. K. and Mehmet, A. 2005. Effects of salt and drought stresses on germination and seedling growth of Pea (Pisum Sativum L.). Turk J. Agric For., 25: 237-242.

Michel, B.E., and Kaufmann, M.R. 1973. The osmotic potential of polyethylene glycol 6000. Plant Physiol., 51: 914-916.

Nandi, S., and Bera, A. K. 1995. Seed Res., 23(2): 125-128.

Rajwinder Kuar, Usha Parmar., Jagmeet Kaur and Bains. 2011. Genotypic difference in germination ability of summer mungbean to water stress using polyethylene glycol. J. of Food 
Legumes. 24(1): 79-82.

Rodriguez, M., Canales, E. and Hidalgo, O.B. 2005. Molecular aspects of abiotic stress in plants. Biotechlogia Aplicada. 22: $1-10$.

Shaheen, R., and Hood-Nowotny, R.C. 2005. Effect of drought and salinity on carbon isotope discrimination in wheat cultivars. Plant Sci. 168: 901- 909. Tuberosa, and Salvi, S. 2006. Genomicsbased approaches to improve drought tolerance of crops. Trends Plant Sci., 11: 405-412.

\section{How to cite this article:}

Swathi, L., D.M. Reddy, P. Sudhakar and Vineela, V. 2017. Screening of Mungbean (Vigna radiata L. Wilczek) Genotypes against Water Stress Mediated through Polyethylene Glycol. Int.J.Curr.Microbiol.App.Sci. 6(10): 2524-2531. doi: https://doi.org/10.20546/ijcmas.2017.610.296 